\title{
THE BENEFIT OF ALTRUISTIC BEHAVIOUR ACHIEVED BY THE OUT POLICY WITH UNMATCHED PROPORTIONAL FEEDBACK GAINS IN A TWO- ECHELON SUPPLY CHAIN
}

\author{
Stephen M. Disney* and Takamichi Hosoda \\ Logistics Systems Dynamics Group, Cardiff Business School, Cardiff University, \\ Aberconway Building, Colum Drive, Cardiff, CF10 3EU, UK. \\ * Corresponding author
}

\begin{abstract}
We study a two echelon supply chain with $A R(1)$ demand and unit replenishment lead-times. Each echelon of the supply chain uses conditional expectation to generate MMSE forecasts. Both echelons use these forecasts inside the "Order-Up-To" policy to generate replenishment orders. We investigate 3 different scenarios: The first is when each echelon aims to minimize their own inventory holding and backlog costs. The second scenario is concerned with an altruistic retailer who is willing and able to sacrifice some of his own performance for the benefit of the total supply chain. He does this by smoothing the demand he places on the manufacturer by using a matched proportional controller in the inventory and WIP feedback loops. The third scenario is concerned with an altruistic retailer with two unmatched controllers. The matched controller case outperforms the traditional case by $14.1 \%$; the unmatched controller case outperforms the matched controller case by $4.9 \%$.
\end{abstract}

Keywords: Supply chains, multi-echelon inventory, order-up-to policy, collaboration, altruistic behavior

\section{INTRODUCTION}

Assuming $A R(1)$ demand, we study a serially linked twoechelon supply chain that exploits a generalized order-upto OUT policy with unmatched (that is, independent), feedback controllers at the first echelon (the retailer) and a traditional OUT policy at the second echelon (the manufacturer). We also assume that minimum mean square error forecasting is used and unit lead-times are present at each echelon.

The benefit from the retailer's altruistic behavior enabled by the generalized OUT policy with unmatched feedback controllers will be investigated, [1]. Each player acts to minimize global inventory costs. To quantify its benefit, this strategy will be compared with other two strategies; 1) a traditional strategy where each player minimizes local inventory costs [2], and 2) an altruistic strategy achieved by the generalized OUT policy with matched feedback controllers [3].

As an indicator of the supply chain performance, we will employ a metric that consists of the sum of the stationary standard deviation of the net inventory levels at each echelon. This is a valid approach when safety stock have been optimized via the newsvendor principle as inventory costs are then linearly related to the standard deviation to the inventory levels, [4]. We also quantify the bullwhip effect in the supply chain.

We reveal the exact analytical expressions of the performance indicators. We highlight that the generalized OUT policy with unmatched controllers enable us to manipulate the dynamics of a supply chain with higher degree of freedom than the generalized OUT policy with matched controllers. Furthermore, we also discuss what kind of information should be shared between these two players to achieve the benefits from the altruistic strategy we highlight herein.

\section{DIFFERENCE EQUATION REPRESENTATION OF THE TWO-ECHELON SUPPLY CHAIN}

We assume the demand faced by the retailer is a mean centered autoregressive stochastic process of the first order. Thus,

$d_{1, t}=\mu_{d}+\rho\left(d_{1, t-1}-\mu_{d}\right)+\varepsilon_{t}$, and the demand faced by the manufacturer in the second echelon is the retailers order,

$d_{2, t}=o_{1, t}$.

In Eq (1), $\rho$ is the autoregressive constant $(-1<\rho<1), d_{t}$ is the demand at time $t$ and $\mu_{d}$ is the average demand. We assume $\mu_{d} \gg 4 \sigma_{d}$ so that the possibility of negative demand is negligible. $\varepsilon_{t}$ is a stochastic white noise process. In Eq (2), $o_{t}$ is the orders at time $t$.

We will also assume that there is a unit replenishment lead-time at each echelon. Additionally, there is a one period, order of events delay. Thus at both echelons (where the first part of the subscript is used to indicate the echelon in question; $x=1$ for the retailer and $x=2$ for the manufacturer), the following inventory balance equation holds,

$n s_{x, t}=n s_{x, t-1}-d_{x, t}+o_{x, t-2}$,

where $n s$ is the net stock (inventory on hand). In each ordering policy we will also need two forecasts of demand. One of these forecasts is the conditional expectation of the demand in the next period and this is used to generate a desired WIP (or pipeline, orders placed but not yet received) target. The other forecast is the conditional expectation of demand in the period after the replenishment order arrives, that is, the forecast of demand in the next, next period. For the retailer these forecasts are

$$
d \text { wip }_{1, t}=E\left[d_{1, t+1}\right]=\rho d_{1, t}
$$$$
\hat{d}_{1, t}=E\left[d_{1, t+2}\right]=\rho^{2} d_{1, t}
$$

However, these two forecasts are considerably more complex for the manufacturer. They are

$$
\begin{aligned}
& d w i p_{2, t}=E\left[o_{1, t+1}\right]=\rho^{3} d_{1, t}+\frac{\rho^{2} d_{1, t}-o_{1, t}}{T w}+\frac{t n s-n s_{1, t}-o_{1, t-1}+\rho d_{1, t}}{T i} \\
& \hat{d}_{2, t}=E\left[o_{1, t+2}\right]=\left(\begin{array}{l}
\left(\rho^{3}+\rho^{4}\right) d_{1, t}+ \\
\frac{1}{T w}\left(\rho^{2} d_{1, t}-o_{1, t}-\frac{\rho^{2} d_{1, t}-o_{1, t}}{T w}-\frac{t n s-n s_{1, t}-o_{1, t-1}+\rho d_{1, t}}{T i}\right)
\end{array}\right)
\end{aligned}
$$

We may use these forecasts in the following difference equation to generate orders (which holds at both 

chain", International Conference on Production Research, July 29th-August 2nd, Valparaiso Chile.

echelons),

$o_{x, t}=\hat{d}_{x, t}+\frac{1}{T i}\left(t n s-n s_{x, t}\right)+\frac{1}{T w}\left(d w i p_{x, t}-o_{x, t-1}\right)$

These last few difference equations (Eqs 6-8) contain some new notation. The first is ths, the target net stock, a time invariant target safety stock that is used to ensure a desired fill-rate or availability of stock is achieved. The other two new terms are $T i$ and $T w$. These are linear feedback gains in the net stock and WIP feedback loops respectively. Feedback gains are a very simple and very well known technique from the field of control theory for manipulating the response of a dynamic system. When $T w=T i=1$ we say then the supply chain consists of two serially linked, "traditional" OUT policies; when $T w=T i$, we say there are "matched controllers; when $T w \neq T i$ we say there are "unmatched controllers".

\section{THE OBJECTIVE FUNCTION}

We will consider minimising the following objective function

$$
J=\sqrt{\operatorname{Var}\left[N S_{1}\right]}+\sqrt{\operatorname{Var}\left[N S_{2}\right]} .
$$

This is an appropriate objective function when there are inventory holding and backlog costs that are linear in the inventory position in cases when tns has been set to the critical fractile to minimize the costs via the newsboy principle. The fact that we have simply added the two standard deviations together also implies that the retailer's inventory holding and backlog costs are as important as the manufacturer's inventory holding and backlog costs.

[3] shows that setting $T i=T w=1$ at the manufacturer (the second echelon) yields a minimum value of $J$ in a given scenario. Therefore, in our two-echelon model, only the first echelon (the retailer) exploits the feedback controllers ( $T i$ and $T w$ ) to manipulate the dynamics of the supply chain The manufacturer simply uses a traditional order-up-to policy with MMSE forecasting to minimise $J$. This is a natural consequence of our objective function, (Eq 9$)$. If the objective function contains bullwhip related costs then this does not hold and the manufacturer should incorporate feedback controller(s) into his replenishment rule. This is outside the scope of this short conference paper. However, we will quantify order variance at both echelons in our model for completeness.

In the rest of the paper we will compare three scenarios:

- The traditional, local optimisation. This scenario considers the case when both the retailer and the manufacturer are solely concerned with minimising their own, local inventory holding and backlog costs. We will study this scenario in section 4.

- The altruistic retailer, global optimisation with matched controllers. This scenario considers the case when the retailer is able and willing to alter his replenishment rule (by tuning $\mathrm{Ti}$ ) in order to minimise the total supply chain costs. We assume in this case that the retailer uses a generalised OUT policy with matched controllers, $T w=T i$. We will study this scenario in section 5 .

- The altruistic retailer, global optimisation with unmatched controllers. We will study this scenario in section 6 and is essentially the same as the previous strategy but with independent, unmatched controllers in the retailer's replenishment rule, that is $T w \neq T i$.

\section{ANALYSIS OF THE TRADITIONAL OUT POLICY SCENARIO; THE LOCAL OPTIMISATION}

Here the retailer uses $T i=T w=1$ and thus the supply chain consists of two serially linked OUT policies with MMSE forecasting. As $T i=T w=1$ there are no stability issues in the supply chain and the variance of the two net stock positions turns out to be

$$
\begin{aligned}
\operatorname{Var}\left[N S_{1}\right] & =\left(2+2 \rho+\rho^{2}\right) \sigma_{\varepsilon}^{2} \\
\operatorname{Var}\left[N S_{2}\right] & =(2+\rho(4+\rho(6+\rho(6+\rho(4+\rho(2+\rho)))))) \sigma_{\varepsilon}^{2} \\
& =\left(2+4 \rho+6 \rho^{2}+6 \rho^{3}+4 \rho^{4}+2 \rho^{5}+\rho^{6}\right) \sigma_{\varepsilon}^{2}
\end{aligned}
$$

These expressions for the variances may, in general, be obtained by a variety of ways, from stochastic analysis [2], via the frequency domain [5], control theory [6], or state space methods [7]. However we will not provide further details here due to space requirements. Figure 1 illustrates the inventory costs (via the standard deviations used in the objective function, Eq (9)) as a function of the autoregressive parameter, $\rho$.

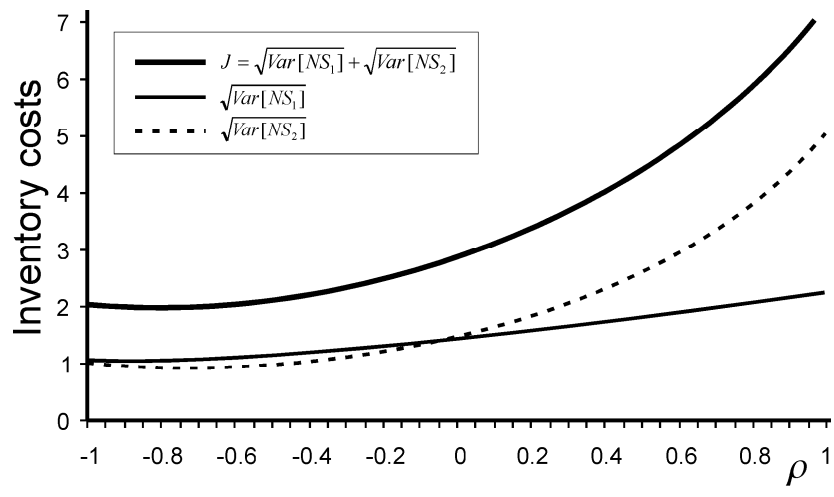

Figure 1: The inventory variances in the traditional supply chain

The variance expressions for the demand and the two order rates are;

$$
\begin{aligned}
& \operatorname{Var}\left[D_{1}\right]=\sum_{t=0}^{\infty}\left(\rho^{t}\right)^{2}=\frac{1}{1-\rho^{2}} \sigma_{\varepsilon}^{2} \\
& \operatorname{Var}\left[O_{1}\right]=\frac{2 \rho\left(\rho^{3}+\rho^{4}-1-\rho\right)-1}{\rho^{2}-1} \sigma_{\varepsilon}^{2} \\
& \operatorname{Var}\left[O_{2}\right]=\frac{2 \rho(\rho-1)(1+\rho)\left(1+\rho^{2}\right)\left(1+\rho+\rho^{2}+\rho^{3}+\rho^{4}\right)-1}{\rho^{2}-1}
\end{aligned}
$$

which have been plotted in Figure 2. Note from Figure 2 that when $\rho>0$ then a bullwhip effect exists as $\operatorname{Var}\left[O_{1}\right]>\operatorname{Var}\left[D_{1}\right]$ and $\operatorname{Var}\left[O_{2}\right]>\operatorname{Var}\left[D_{1}\right]$.

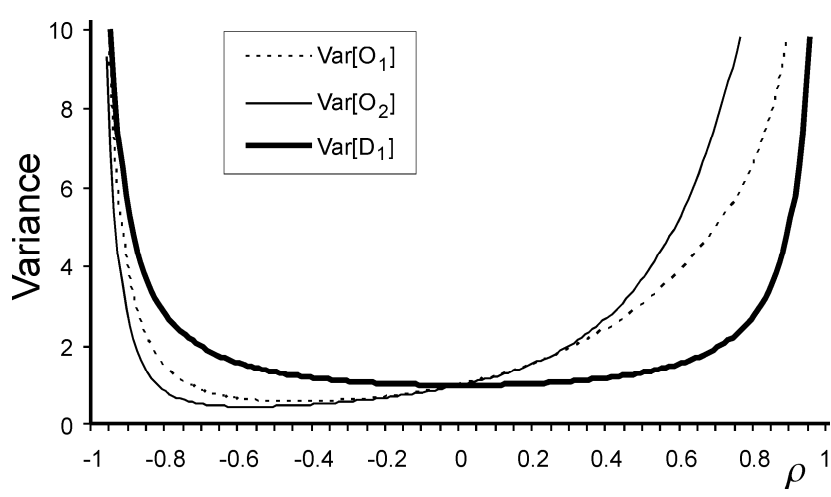

Figure 2: Order variances in the traditional supply chain

\section{ANALYSIS OF THE GENERALISED OUT POLICY SCENARIO WITH MATCHED CONTROLLERS}

By setting $T w=T i$, at the retailer we have matched feedback 

chain", International Conference on Production Research, July 29th-August $2^{\text {nd }}$, Valparaiso Chile.

controllers. This yields a new set of variance ratio formulas. These are:

$\operatorname{Var}\left[N S_{1}\right]=\frac{T i\left(2+T i(1+p)^{2}\right)-1}{2 T i-1} \sigma_{\varepsilon}^{2}$

$\operatorname{Var}\left[N_{2}\right]=\frac{\left(\begin{array}{l}(1+\rho)^{2}-4 T i(1+\rho)^{2}+2 T i^{3} \rho^{2}(1+\rho)(3+2 \rho)- \\ T i^{2}(1+\rho)^{2}\left(2 \rho^{2}-5\right)+T i^{4} \rho^{4}(2+\rho(2+\rho))\end{array}\right)}{T i^{4}} \sigma_{\varepsilon}^{2}$

From Eq (15) we can see that the valid range of $\mathrm{Ti}$ to ensure stability is $0.5<T i<\infty$. We may use these variance ratios in the objective function (Eq 9) and determine the $T i$ that minimises the objective function, $T i *$. Analytically this appears to be very difficult to achieve. However, using numerical techniques is considerably less complex and results in the following graphical relationship, see Figure 3.

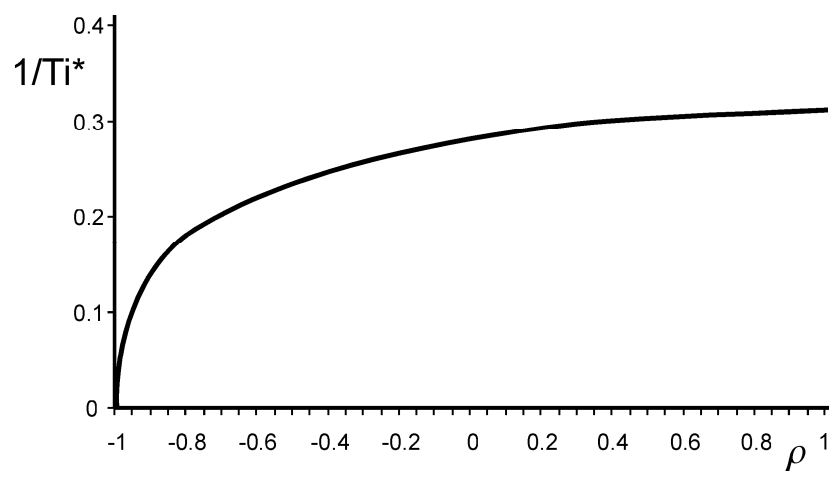

Figure 3. The optimal Ti when matched controllers exist with an altruistic retailer

Using this optimal $T i$ inside the objective function results in Figure 4 which describes the minimised inventory costs in our supply chain.

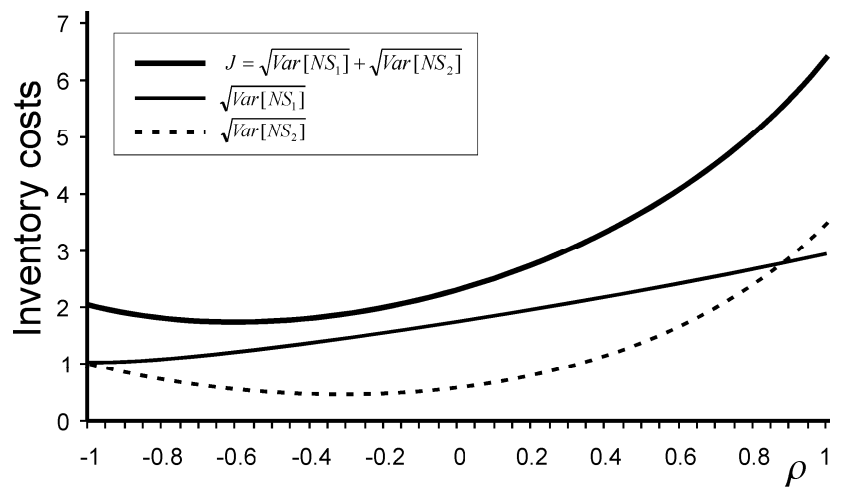

Figure 4. The objective function with matched controllers at $T i=T i^{\star}$ with an altruistic retailer

The general expressions for the order variances are given by Eqs (17) and (18).

$$
\begin{aligned}
& \operatorname{Var}\left[O_{1}\right]=\frac{\left(\begin{array}{l}
T i+\rho+T i \rho+2 T i \rho^{2}+ \\
2(T i-1) \rho^{3}+2(T i-2) T i \rho^{4}-2 T i^{2} \rho^{5}
\end{array}\right)}{(2 T i-1)(T i(\rho-1)-\rho)\left(\rho^{2}-1\right)} \sigma_{\varepsilon}^{2} \\
& \left(2 T i(\rho-1)(1+\rho)^{3}(7 \rho-1)-2(\rho-1) \rho(1+\rho)^{3}+\right. \\
& 2 T i^{2}(\rho-1)(1+\rho)^{3}\left(6-20 \rho+\rho^{3}\right)+2 T i^{7} \rho^{4}\left(1+\rho-2 \rho^{3}-2 \rho^{4}+2 \rho^{6}\right)- \\
& 2 T i^{3}(\rho-1)(1+\rho)^{2}(14+\rho(\rho(\rho(5+\rho(5+\rho))-30)-15))+ \\
& 2 T i^{4}(\rho-1)(1+\rho)^{2}(15+\rho(\rho(\rho(9+\rho(9+5 \rho))-26)-6))+ \\
& T i^{6}\left(1+\rho\left(1-2(\rho-1) \rho\left(5+\rho\left(10+\rho\left(4+\rho\left(5 \rho^{3}+3 \rho^{4}-5-7 \rho\right)\right)\right)\right)\right)\right)+ \\
& \operatorname{Var}\left[\mathrm{O}_{2}\right]=\frac{\left(\operatorname{Ti}^{5}\left(12+\rho\left(13+2 \rho\left(\rho\left(\rho\left(26+\rho\left(22+\rho\left(\rho^{3}+\rho^{4}-5-15 \rho-8 \rho^{2}\right)\right)\right)-15\right)-20\right)\right)\right)\right.}{\left(T i^{5}(2 T i-1)(T i(\rho-1)-\rho)(\rho-1)(1+\rho)\right)} \sigma^{2}
\end{aligned}
$$

When $\mathrm{Ti}$ has been set to $\mathrm{Ti}^{*}$ then the order variances can be plotted as shown in Figure 5. Comparison of Figures 2 and 5 shows that the altruistic contribution of the retailer results in a smoothing of the retailers order variance. Thus, if the retailer incurs some bullwhip related costs in his retail, warehousing or transportation activities, then he may in fact, be even more willing to use the proportional feedback controller to minimize costs at the supplier than this stylized analysis suggests.

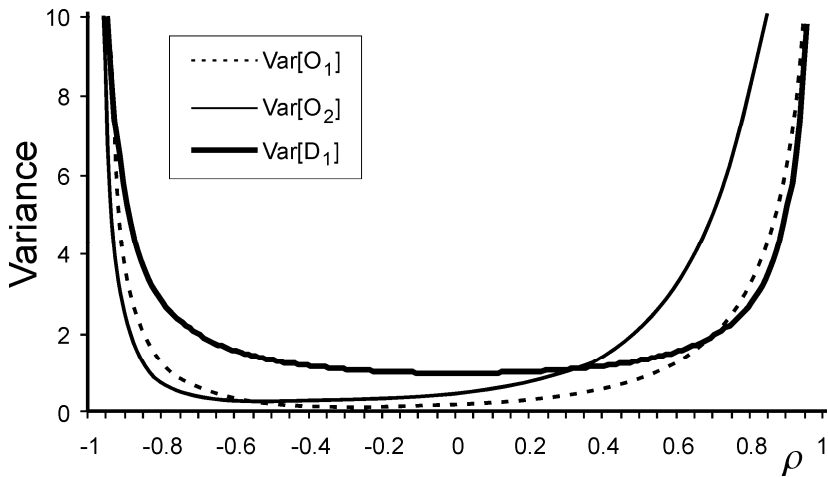

Figure 5. The demand and order variances with matched controllers at $T i=T i *$ with an altruistic retailer

\section{ANALYSIS OF THE GENERALISED OUT POLICY SCENARIO WITH UN-MATCHED CONTROLLERS}

As there are two unmatched controllers then there is a need to conduct a stability analysis at the retailer. There is no need to consider such issues at the manufacturer as here $T i=T w=1$ which results in a stable system. Thus the analysis for the generalised OUT policy with unmatched controllers consists of a two stage approach.

\subsection{Stability analysis}

Stability can be readily investigated via transfer functions and we will exploit Jury's Inners approach to conduct the analysis, [8]. This transfer function of the retailers order is

$$
\frac{O_{1}(z)}{\varepsilon(z)}=\frac{z^{2}\left(T i(z-1) \rho+T w\left(z+T i(z-1) \rho^{2}\right)\right)}{(T w+T i(z-1)(1+T w z))(z-\rho)} \text {. }
$$

It is known that stability only depends upon feedback loops and thus we may ignore the feed-forward autoregressive term. Setting $\rho=0$ and simplifying results in

$$
\frac{O_{1}(z)}{\varepsilon(z)}=\frac{T w z^{2}}{T w+T i(z-1)(1+T w z)}
$$

Jury's stability test requires us to expand out the denominator and collect together powers of $z$.

$A(z)=T w-T i+z(T i(1-T w))+z^{2} T i T w$

The first part of Jury's stability test is to ensure that $A(1)>0$ where $A(1)=\left.A(z)\right|_{z \rightarrow 1}$. Thus it follows that $T w>0$.

The second stage of Jury's test is that $(-1)^{n} A(-1)>0$. This is true iff,

$$
T i<\frac{T w}{2(1-T w)} \text {. }
$$

The third and final stage of Jury's stability test is that certain matrices of the co-efficient of the denominator of the systems transfer function are positive innerwise. Because our transfer is only of second order, this criteria easily reduces to the fact that

$T i>\frac{T w}{1+T w}$ 
Disney, S.M. and Hosoda, T. (2007) "The benefit of altruistic behaviour achieved by the OUT policy with unmatched proportional feedback gains in a two-echelon supply chain", International Conference on Production Research, July 29th-August 2nd, Valparaiso Chile.

$T i>\frac{T w}{1-T w}$.

Numerical investigation reveals that (24) is non critical as it is entirely encompassed by (22). It is interesting to note that the stability bound in first step of Jury's test results in $T w>0$, but (23) shows us that $T w$ can in fact be negative. Careful investigation shows that the unstable region of $T w$, becomes stable when $T w<-1$. For confirmation a more direct stability test is given in [9] and results in $T w>0$, Eq 22 and Eq 23. The redundant condition produced by Jury's Inners Test is not generated. Figure 6 illustrates the stability region.

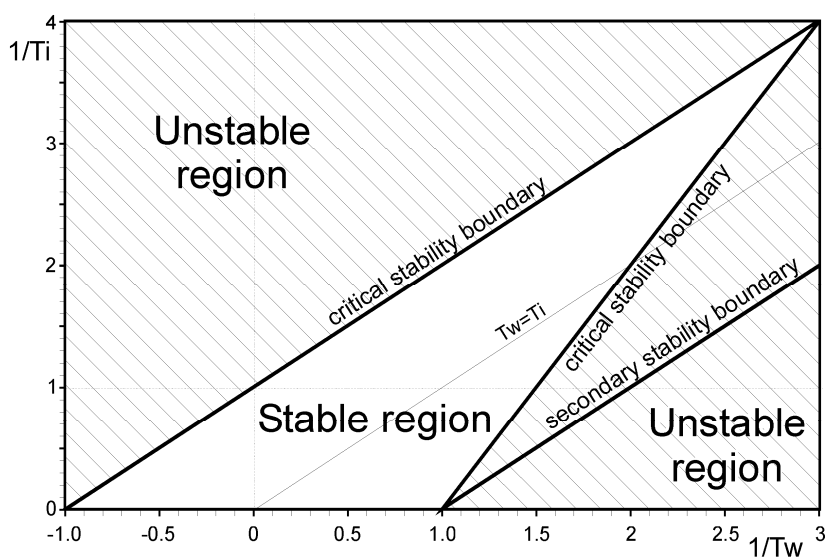

Figure 6: The stability boundary for the generalized OUT policy with unit lead-times

\subsection{Variance analysis}

The variance of the retailer's net stock is given by,

$\operatorname{Var}\left[N S_{1}\right]=\frac{T i^{2}\left(T w+T i(T w-1)(1+T w+T w \rho)^{2}+T w^{2}\left(T w+2 \rho+T w \rho^{2}\right)\right)}{T w(2 T i(T w-1)+T w)(T i+(T i-1) T w)} \sigma_{\varepsilon}^{2}$

The variance of the manufacturer's net stock is given by,

$\operatorname{Var}\left[N S_{2}\right]=\left(\rho^{2}+\rho^{3}+\frac{\rho}{T w}-\frac{\rho}{T w^{2}}-\frac{1}{T i T w}+\frac{2+\rho}{T i}\right)^{2} \sigma_{\varepsilon}^{2}+\left(\rho^{2}+\frac{\rho}{T w}+\frac{1}{T i}\right)^{2} \sigma_{\varepsilon}^{2}$

Using these variance ratios in the objective function we may find the optimal values of the unmatched feedback controllers. Again, analytically this is very difficult, but numerical techniques do exist and they result in values for $T i$ and $T w$ as shown in the Figure 7 . Figure 7 contains some very remarkable features. For $\rho<-0.25734$ both $\mathrm{Ti}^{*}$ and $T w^{*}$ are positive. However as there are two local optimums in the solution space near $\rho \approx-0.25734$ then the optimal $\mathrm{Ti}^{*}$ and $T w^{*}$ is discontinuous in $\rho$. Interestingly, the optimal $T w^{*}$ is negative for $\rho>-0.25734$.

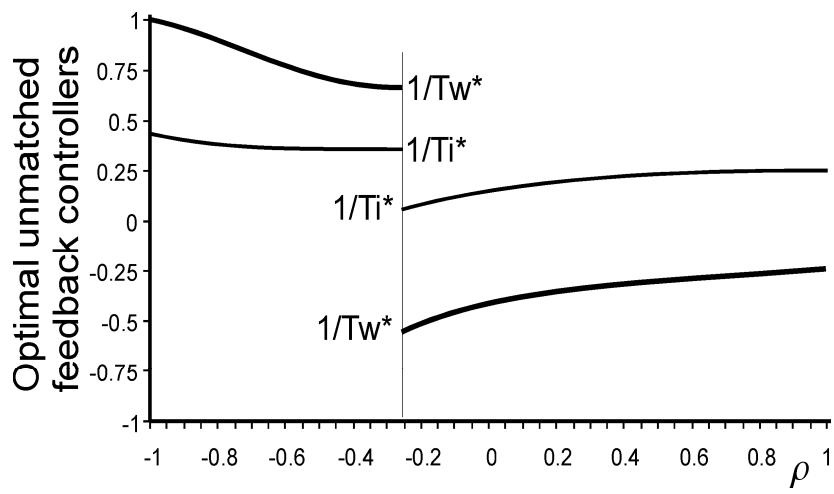

Figure 7 . Tuning the unmatched feedback controllers to minimise supply chain inventory costs

Using these values in the objective function we may illustrate the inventory costs as shown in Figure 8 . Here we can see the impact of the discontinuous $\mathrm{Ti}^{*}$ and $\mathrm{Tw}^{*}$.

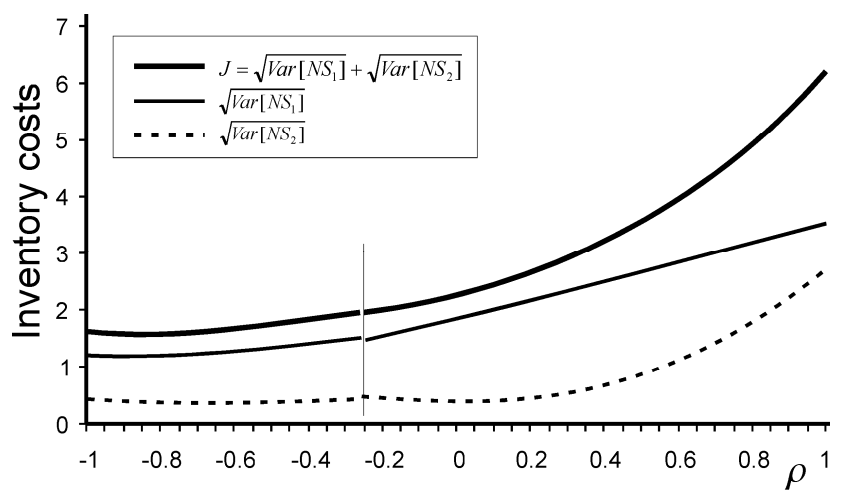

Figure 8. The objective function with unmatched controllers with an altruistic retailer

Returning now to the impact of altruistic retailer with unmatched controllers on the variances of the order rates we have;

$\operatorname{Var}\left[O_{1}\right]=\frac{4 T w\left(\begin{array}{l}T w^{3} \rho^{2}+2 T i^{3}(T w-\rho)(\rho-1) \rho^{2}(1+T w \rho)^{2}+T i T w^{2}\left(\begin{array}{l}\rho(2 \rho(\rho-2)-1)+ \\ T w(1+\rho(\rho-1))\left(2 \rho^{2}-1\right)\end{array}\right) \\ T i^{2} T w\left(\begin{array}{l}\rho+\rho^{2}(3+2 \rho(\rho-2))+T w(\rho-1)\left(2 \rho^{2}-1\right)(1+\rho(2 \rho-1))+ \\ T w^{2}(1+\rho)\left(2 \rho^{2}(\rho-1)(1+\rho(\rho-1))-1\right)\end{array}\right)\end{array}\right)}{\left(\begin{array}{l}(2 T i(T w-1)+T w)(T i+(T i-1) T w)\left(\sqrt{T i}(T w(\rho-2)-\rho)-\rho \sqrt{T i(1+T w)^{2}-4 T w^{2}}\right) \\ \left(\sqrt{T i}(T w(\rho-2)-\rho)+\rho \sqrt{T i(1+T w)^{2}-4 T w^{2}}\right)\left(\rho^{2}-1\right)\end{array}\right)} \sigma_{s}^{2}$

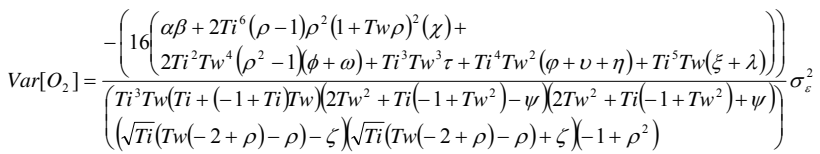

where we have used the following substitutions as the formula is rather complex.

$\psi=\sqrt{T i}(T w-1) \sqrt{T i(1+T w)^{2}-4 T w^{2}}, \zeta=\rho \sqrt{T i(1+T w)^{2}-4 T w}$

$\alpha=2 T w^{7} \rho^{2}\left(1-\rho^{2}\right)(T w(2+\rho)-1)-2 T i T w^{5}\left(1+\rho^{2}\right)$

$\beta=\left(\begin{array}{l}\rho^{2}+T w \rho\left(\rho-1-4 \rho^{2}\right)+T w^{3}(\rho-2)\left(\rho+\rho^{2}-1\right)\left(1+\rho+\rho^{2}\right) \\ +T w^{2}(2 \rho-1)(1+\rho(\rho(2+\rho)-1))\end{array}\right)$

$\left(2 T w^{2}(1+2 \rho)\left(-1+\rho^{2}\right)^{2}-2 T w^{4}(-1+\rho)(1+\rho)\left(-1+\rho^{2}(2+3 \rho)\right)+\right.$

$\chi=T w^{6}\left(-1+2 \rho\left(-1-\rho+\rho^{3}+\rho^{4}\right)\right)+T w^{5}\left(2+\rho\left(3+2 \rho^{2}(1+\rho)\left(-2+\rho^{2}\right)\right)\right)-$

$2 T w(-1+\rho)(1+\rho)^{2}(-1+2 \rho)-$

$2 T w^{3}(-1+\rho) \rho(1+\rho)(2+\rho)(-1+(-1+\rho) \rho)+2 \rho\left(-1+\rho^{2}\right)$

$\phi=\rho(2 \rho(2-\rho)-1)+T w^{4}(1+\rho)\left(1+\rho(1+\rho)\left(1+2 \rho+\rho^{3}+\rho^{4}\right)\right)$

$(2(\rho-1) \rho(1+\rho)(\rho(8+\rho(4 \rho-11))-2)+$

$2 T w(\rho-1)(1+\rho)(\rho(2+\rho)(7+\rho(10 \rho-13))-2)-$

$\varphi=4 T w^{2}\left(3+\rho\left(\rho^{2}\left(12+\rho(1+\rho)(2 \rho-3)\left(2+\rho^{2}\right)\right)-9\right)\right)+$

$T w^{5}\left(\rho\left(2 \rho\left(30+\rho\left(8+\rho\left(15 \rho^{3}+11 \rho^{4}+\rho^{5}-3 \rho^{6}-4 \rho^{7}-28-19 \rho\right)\right)\right)-3\right)-16\right)$

$\left(2 T w^{2}(\rho-1) \rho(1+\rho)\left(8+\rho\left(\rho\left(2-\rho+5 \rho^{3}\right)-10\right)\right)+\right.$

$2 T w^{4} \rho\left(4+\rho\left(\rho\left(\rho\left(9+\rho(1-2(\rho-1) \rho)^{2}\right)-9\right)-5\right)\right)-$

$\xi=2 T w(\rho-1) \rho(1+\rho)(3 \rho-4)(3 \rho-1)-$

$2 T w^{3}\left(\rho^{4}-4 \rho+2 \rho^{5}+7 \rho^{6}-3 \rho^{7}-8 \rho^{8}+5 \rho^{9}\right)+$

$2 \rho^{2}\left(4+\rho\left(\rho-7+7 \rho^{2}-5 \rho^{3}\right)\right)$

$\left(T w^{6}(\rho-1)\left(\rho\left(9+2 \rho^{2}\left(\rho(1+\rho)\left(3+\rho\left(7-2 \rho-3 \rho^{3}+\rho^{4}\right)\right)-7\right)\right)-1\right)-\right.$

$\lambda=T w^{7}(1+\rho)\left(2 \rho^{2}\left(\rho\left(2+\rho\left(4+\rho\left(4+\rho\left(-3+\rho\left(-4+\rho\left(-1+\rho+2 \rho^{2}\right)\right)\right)\right)\right)\right)-5\right)-1\right)+$

$T w^{5} \rho\left(\rho\left(5+2 \rho\left(9+\rho\left(\rho\left(3+\rho\left(11+\rho\left(\rho\left(2 \rho^{2}+5 \rho^{3}-9-2 \rho\right)-5\right)\right)\right)-9\right)\right)\right)-17\right)$ 

chain", International Conference on Production Research, July 29th-August 2nd, Valparaiso Chile.

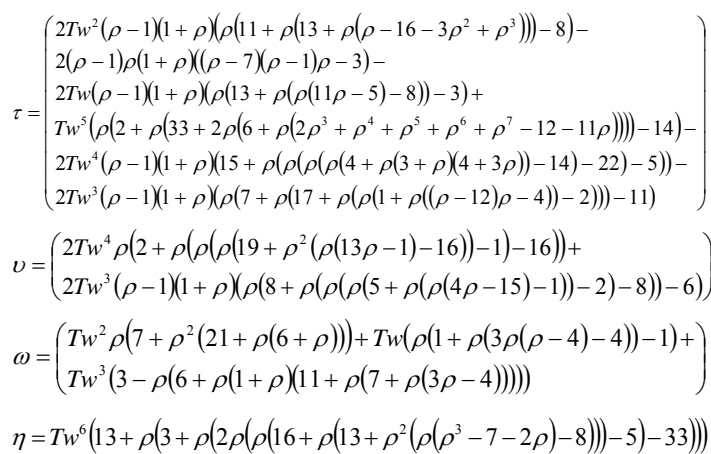

These expressions are plotted in Figure 9.

(37)

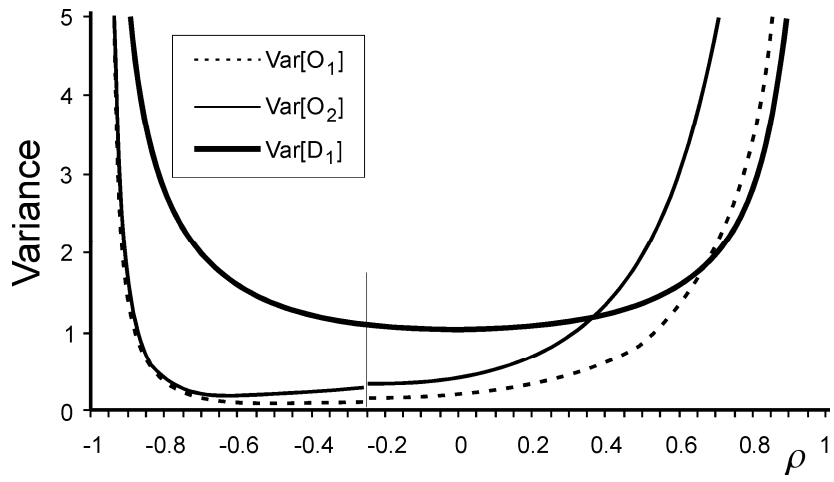

Figure 9. The order variances with unmatched controllers (of $T i=T i^{*}$ and $T w=T w^{*}$ ) at with an altruistic retailer

Table 1. Enumeration of the three supply chain scenarios

\begin{tabular}{|c|c|c|c|c|c|c|c|c|c|c|c|c|c|c|}
\hline \multirow[b]{2}{*}{$\rho$} & \multicolumn{3}{|c|}{ Traditional Supply Chain, $\mathrm{Ti}=\mathrm{Tw}=1$} & \multicolumn{5}{|c|}{ Altruistic Retailer with Matched Controllers } & \multicolumn{6}{|c|}{ Altruistic Retailer with Unmatched Controllers } \\
\hline & $\begin{array}{c}\text { Retailers } \\
\text { Inventory } \\
\text { Cost }\end{array}$ & $\begin{array}{c}\text { Man. } \\
\text { Inventory } \\
\text { Cost }\end{array}$ & $\begin{array}{c}\text { Total } \\
\text { Inventory } \\
\text { Costs }\end{array}$ & $T W^{*}=T_{i}{ }^{*}$ & $\begin{array}{c}\text { Retailers } \\
\text { Inventory } \\
\text { Cost }\end{array}$ & $\begin{array}{c}\text { Man. } \\
\text { Inventory } \\
\text { Cost }\end{array}$ & $\begin{array}{c}\text { Total } \\
\text { Inventory } \\
\text { Costs }\end{array}$ & $\begin{array}{l}\text { \% benefit } \\
\text { above } \\
\text { traditional } \\
\text { supply chain }\end{array}$ & $\mathrm{Ti}^{*}$ & $T w^{*}$ & $\begin{array}{c}\text { Retailers } \\
\text { Inventory } \\
\text { Cost }\end{array}$ & $\begin{array}{l}\text { Man. } \\
\text { Inventory } \\
\text { Cost }\end{array}$ & $\begin{array}{c}\text { Total } \\
\text { Inventory } \\
\text { Costs }\end{array}$ & $\begin{array}{l}\text { \% benefit } \\
\text { above } \\
\text { matched } \\
\text { supply } \\
\text { chain }\end{array}$ \\
\hline-1 & 1 & 1 & 2 & 99999 & 1 & 1 & 2 & 0 & 2.2916 & 1 & 1.210608 & 0.43637 & 1.64698 & 17.650818 \\
\hline-0.9 & 1.004987 & 0.927826 & 1.93281 & 7.0522 & 1.015004 & 0.836012 & 1.85101 & 4.23202052 & 2.5032 & 1.0538 & 1.212717 & 0.36862 & 1.58134 & 14.568868 \\
\hline-0.8 & 1.019803 & 0.901767 & 1.92157 & 5.4967 & 1.058742 & 0.703698 & 1.76244 & 8.28125428 & 2.2592 & 1.12469 & 1.222485 & 0.34969 & 1.57219 & 10.795020 \\
\hline-0.7 & 1.044030 & 0.907694 & 1.95172 & 4.8776 & 1.115600 & 0.608615 & 1.72421 & 11.6568244 & 2.7322 & 1.21156 & 1.245786 & 0.35458 & 1.60038 & 7.1824312 \\
\hline-0.6 & 1.077033 & 0.934631 & 2.01166 & 4.5240 & 1.186125 & 0.540233 & 1.72635 & 14.1825429 & 2.7945 & 1.30595 & 1.283655 & 0.36894 & 1.6526 & 4.2723559 \\
\hline-0.5 & 1.118034 & 0.976281 & 2.09431 & 4.2717 & 1.266789 & 0.494717 & 1.76150 & 15.8910170 & 2.8298 & 1.39529 & 1.334536 & 0.38756 & 1.7221 & 2.2372178 \\
\hline-0.4 & 1.166190 & 1.030543 & 2.19673 & 4.0655 & 1.354407 & 0.469610 & 1.82401 & 16.9668312 & 2.8380 & 1.46523 & 1.396672 & 0.40978 & 1.80646 & 0.9628588 \\
\hline-0.3 & 1.220655 & 1.098303 & 2.31895 & 3.8898 & 1.446924 & 0.463397 & 1.91032 & 17.6215702 & 2.8195 & 1.50119 & 1.468354 & 0.43780 & 1.90616 & 0.2178938 \\
\hline-0.2 & 1.280624 & 1.182294 & 2.46291 & 3.7443 & 1.543644 & 0.475552 & 2.01919 & 18.0161371 & 11.318 & -1.9466 & 1.532943 & 0.45499 & 1.98794 & 1.5480031 \\
\hline-0.1 & 1.345362 & 1.286227 & 2.63158 & 3.6294 & 1.644620 & 0.507137 & 2.15175 & 18.2335213 & 8.0763 & -2.16987 & 1.68466 & 0.41019 & 2.09486 & 2.6443885 \\
\hline 0 & 1.414213 & 1.414213 & 2.82842 & 3.5414 & 1.749812 & 0.561211 & 2.31102 & 18.2929485 & 6.6096 & -2.39687 & 1.8387 & 0.39577 & 2.23447 & 3.3125049 \\
\hline 0.1 & 1.486606 & 1.570484 & 3.05709 & 3.4738 & 1.869447 & 0.631878 & 2.50132 & 18.1795635 & 5.7847 & -2.6212 & 1.994565 & 0.41342 & 2.40799 & 3.7314174 \\
\hline 0.2 & 1.562049 & 1.759289 & 3.32134 & 3.4201 & 1.970832 & 0.756007 & 2.72684 & 17.8994008 & 5.2690 & -2.83001 & 2.153179 & 0.46502 & 2.6182 & 3.9840359 \\
\hline 0.3 & 1.640121 & 1.984940 & 3.62506 & 3.3762 & 2.085404 & 0.907326 & 2.99273 & 17.4433165 & 4.9208 & -3.01765 & 2.314919 & 0.55435 & 2.86928 & 4.1251672 \\
\hline 0.4 & 1.720465 & 2.251883 & 3.97234 & 3.3394 & 2.202038 & 1.1.101507 & 3.30354 & 16.8364639 & 4.6693 & -3.18779 & 2.479179 & 0.6869 & 3.16611 & 4.1602403 \\
\hline 0.5 & 1.802775 & 2.564786 & 4.36756 & 3.3080 & 2.320380 & 1.343665 & 3.66404 & 16.1077385 & 4.4777 & -3.34643 & 2.645139 & 0.86864 & 3.51379 & 4.1009575 \\
\hline 0.6 & 1.886796 & 2.928579 & 4.81537 & 3.2805 & 2.440127 & 1.639021 & 4.07914 & 15.2890968 & 4.3265 & -3.49769 & 2.812214 & 1.10518 & 3.9174 & 3.9651666 \\
\hline 0.7 & 1.972308 & 3.348462 & 5.32077 & 3.2561 & 2.561007 & 1.992942 & 4.55395 & 14.4118412 & 4.2041 & -3.64383 & 2.980042 & 1.40206 & 4.38211 & 3.7734610 \\
\hline 0.8 & 2.059126 & 3.829869 & 5.88899 & 3.2340 & 2.682787 & 2.410940 & 5.09372 & 13.5043066 & 4.1033 & -3.78616 & 3.148372 & 1.76476 & 4.91314 & 3.5453700 \\
\hline 0.9 & 2.147091 & 4.378447 & 6.52553 & 3.2137 & 2.805271 & 2.898642 & 5.70391 & 12.5908988 & 4.0187 & -3.92556 & 3.317006 & 2.19882 & 5.51583 & 3.2973641 \\
\hline 1 & 2.236067 & 5 & 7.23606 & 3.1947 & 2.928303 & 3.461763 & 6.39006 & 11.6914462 & 3.9468 & -4.06275 & 3.485779 & 2.70988 & 6.19566 & 3.0422908 \\
\hline & & & & & & & Average> & 14.1585114 & & & & & Average > & 4.9103729 \\
\hline
\end{tabular}

\section{NUMERICAL INVESTIGATIONS}

In order to highlight the benefit of the unmatched controllers with altruistic retailer we will now enumerate the inventory costs and order variances for a range of values in the autoregressive demand parameter. This is shown in Table 1.

We can see that if the retailer is able to alter his replenishment rule to incorporate matched feedback controllers then total supply chain inventory costs may be reduced by as much as $14 \%$ when compared to a traditional supply chain. However, if the retailer is will to go even further and use appropriately tuned unmatched controllers, then a further $4.9 \%$ reduction in total supply chain inventory costs may be gained.

\section{CONCLUSIONS}

The unmatched controller generalised OUT policy dominates the matched controller case with an altruistic retailer who is concerned with minimising the global supply chain inventory costs. The benefit appears to be approximately $5 \%$ reduction in the inventory holding and backlog costs. Closer inspection reveals that the altruistic contribution of the retailer, in the unmatched case, is even higher than in the matched case. However, the rewards are even higher when compared to the traditional supply chain where members are only concerned with their local inventory holding and backlog costs as the unmatched controller case is $18.5 \%$ better, on the average.

In order to gain this advantage the first echelon needs to be able to understand the manufacturer's cost structure, the demand signal and the lead-times in the supply chain and then alter the structure of his replenishment rules. This is, indeed, a very complex task and we imagine that it will take considerable industrial engineering efforts to achieve. Even if this could technically be done then the manufacturer has to understand and use market place information and be willing to share some of the economic benefit with his customer. Otherwise, the retailer will have no incentive to make the altruistic contribution and smooth his replenishment orders. Of course, we have also assumed a linear system exists, and thus all unmet demand has been backordered and the statistical properties of the demand signal are time invariant.

As a final point, the analysis therein is very complex and rather ugly. Recent work in [10], suggests that much more 
elegant results can be found by exploiting the "full-statefeedback" controller, a technique advocated by modern control theory. This will be explored in future research.

\section{REFERENCES}

[1] Disney, S.M., Farasyn, I., Lambrecht, M., Towill, D.R., and van de Velde, W., (2006), "Dampening variability by using smoothing replenishment rules", Under review at the European Journal of Industrial Engineering.

[2] Hosoda, T. and Disney, S.M. (2006a) "On variance amplification in a three-echelon supply chain with minimum mean square error forecasting", OMEGA: The International Journal of Management Science 34(4), 344-358.

[3] Hosoda, T. and Disney, S.M. (2006b) "The governing dynamics of supply chains: The impact of altruistic behaviour", Automatica 42(8), 1301-1309.

[4] Disney, S.M., Lambrecht, M., Towill, D.R. and Van de Velde, W., (2006), "The Value of Coordination in a Two Echelon Supply Chain: Sharing information, policies and parameters", Accepted for publication in IIE Transactions.

[5] Dejonckheere, J., Disney, S.M., Lambrecht, M.R. and Towill, D.R., (2004), "The impact of information enrichment on the bullwhip effect in supply chains: A control engineering perspective", European Journal of Operational Research 153(3), 727-750.

[6] Disney, S.M. and Towill, D.R., (2003) "On the bullwhip and inventory variance produced by an ordering policy", OMEGA: The International Journal of Management Science 31(3), 157-167.

[7] Gaalman, G. and Disney, S.M., (2006) "State space investigation of the bullwhip problem with $\operatorname{ARMA}(1,1)$ demand processes", International Journal of Production Economics 104(2) 327-339.

[8] Jury, E.I. (1974) "Inners and the stability of dynamic systems, John Wiley and Sons, New York.

[9] Disney, S.M. (2007) "Supply chain aperiodicity, bullwhip and stability analysis with Jury's Inners", Under review at IMA Journal of Management Mathematics.

[10] Gaalman, G. and Disney, S.M., (2007), "On bullwhip in a family of order-up-to policies with $\operatorname{ARMA}(2,2)$ demand and arbitrary lead-times", Accepted for publication in the International Journal of Production Economics. 